\title{
Uncertainty analysis of a radiative transfer model using Monte Carlo method within $280-2500 \mathrm{~nm}$ region
}

\author{
Giorgio Belluardo $^{\mathrm{a}, \mathrm{d}, *}$, Grazia Barchi ${ }^{\mathrm{a}}$, Dietmar Baumgartner ${ }^{\mathrm{b}}$, Marcus Rennhofer ${ }^{\mathrm{c}}$, \\ Philipp Weihs ${ }^{\mathrm{d}}$, David Moser ${ }^{\mathrm{a}}$ \\ a Institute for Renewable Energy, EURAC Research, Viale Druso 1, 39100 Bolzano, Italy \\ ${ }^{\mathrm{b}}$ Kanzelhöhe Observatory for Solar and Environmental Research, University of Graz, Kanzelhöhe 19, 9521 Treffen am Ossiacher See, Austria \\ ${ }^{\mathrm{c}}$ Energy Department, AIT Austrian Institute of Technology GmbH, Giefinggasse 2, 1210 Vienna, Austria \\ ${ }^{\mathrm{d}}$ Institute of Meteorology, University of Natural Resources and Life Sciences, Peter-Jordan-Straße 82, 1190 Vienna, Austria
}

Received 13 October 2015; received in revised form 23 February 2016; accepted 25 March 2016

Communicated by: Associate Editor David Renne

\begin{abstract}
Radiative transfer models (RTM) are used to calculate spectral and broadband irradiance, given a set of input parameters that are representative of the atmospheric state. While many studies exist on their accuracy, there is still a research gap in the assessment of their uncertainty, due to the nonlinear and not differentiable nature of the Radiative Transfer Equation, which is the core of a RTM. This study evaluates the uncertainty of both spectral and broadband irradiance calculated with the radiative transfer model SDISORT implemented in the tool UVSPEC within the range 280-2500 nm. A set of input values representing the atmospheric state at Kanzelhöhe Observatory (Austria) site at 10:00 on April 25th, 2013 is taken as reference and a Monte Carlo technique is used to propagate the uncertainty of input parameters to the model output. Both the effects of single input parameter uncertainty and of their combination are evaluated, as well as the influence of the deviation of input values from the reference set. Results show that ozone column is an important source of uncertainty in the UV-B region, while the uncertainties of Angström aerosol turbidity coefficient and extraterrestrial spectrum affect the whole spectral range. Considering a reasonable variability range for all involved input parameters, the overall uncertainty of broadband global horizontal irradiance is between $2.9 \%$ and $5.9 \%$. These values are higher, but still comparable, to typical uncertainty values of outdoor-deployed spectroradiometers.
\end{abstract}

(c) 2016 Elsevier Ltd. All rights reserved.

Keywords: Radiative transfer model; Uncertainty evaluation; Spectral irradiance

\section{Introduction}

A reliable assessment of solar resource is important for the development of the renewable energy sector. The uncertainty in modelled or measured irradiance values

\footnotetext{
* Corresponding author at: Institute for Renewable Energy, EURAC Research, Viale Druso 1, 39100 Bolzano, Italy. Tel.: +39 471 055626; fax: +39471055699.

E-mail address: giorgio.belluardo@eurac.edu (G. Belluardo).
}

propagates into models for radiation and energy predictions, performance estimators for energy systems, degradation assessment tools. The attractiveness for investments on solar energy projects is therefore dependent upon the quality of broadband and spectral data.

Simulation of irradiance is useful when measurements are not available at a certain site, or information about the irradiance distribution and variability on a certain area is needed. In order to satisfy these purposes, nowadays 
many tools have been developed that differ on the spectral region resolved, computation algorithm, geometrical schematization, licence type etc. An overview and classification of the most commonly used tools is given by Myers (2005) and by Seidel et al. (2010).

Many authors have reported about accuracy of simulation tools by comparing broadband or spectral irradiance measurements with simulations results, especially under clear-sky conditions (Gueymard, 1995; Myers, 2005; Ding et al., 2011; Clough et al., 2005). Other authors have focused on the estimation of uncertainty of parameters (aerosols, water vapor, ozone etc.) used as input to simulation tools (Gueymard, 2004; Xia et al., 2007; Holben et al., 2001; Perez-Ramirez et al., 2014; Schuster et al., 2006; Toledano et al., 2007; Dubovik et al., 2000; Andrews et al., 2006). However, few studies exist on how much the input uncertainty propagates into the simulation tools generating uncertainty in the output. Among these, Weihs and Webb (1997) and Román et al. (2014a), Román et al. (2014b) calculate the contribution of each input quantity $x$ by performing simulations of broadband irradiance at $x-e$ and $x+e$, where $x$ is a reference value and $e$ is the combined uncertainty associated to $x$, and assuming their difference with the reference output as the output uncertainty. The two mentioned studies differ on the method used to combine the uncertainties of the different input parameters: Weihs and Webb (1997) sum up these single uncertainty contributions, Román et al. (2014b) perform all possible combinations of input parameters to find the one giving the highest output uncertainty. This way, possible correlations between input parameters are properly taken into account. Another method is proposed by Cordero et al. (2007): using Monte Carlo technique, it is possible to calculate the output uncertainty due to the effect of a single input parameter or of the combination of more input parameters by calculating statistics on a series of $N \gg 1$ randomly generated spectra. This way, the analysis can easily be performed both on broadband and spectral model output irradiance. Nevertheless, the analysis performed by Cordero et al. (2007) is limited to the UV region $(280 \mathrm{~nm}$ to $400 \mathrm{~nm})$. The main objective of the present work is to extend the analysis of Cordero et al. (2007) to a broader spectral region $(280 \mathrm{~nm}$ to $2500 \mathrm{~nm})$. The usefulness is twofold: investigating uncertainty propagation at wavelengths that are important (a) for photovoltaic sensitivity to sunlight, and therefore concur to the generation of current, (b) for the sensitivity of the available spectroradiometers, in order to add information on uncertainty when comparing spectral simulations and measurements. The Radiative Transfer Model (RTM) used in the investigation is SDISORT implemented in the tool UVSPEC, and simulations are performed under clear-sky conditions. The paper is structured as follows: Section 2 introduces the RTM SDISORT, the tool UVSPEC and the uncertainty propagation method based on Monte Carlo technique. Section 3 focuses on the obtained results. In particular, Section 3.1 identifies the contributions of each input parameter to SDISORT output uncertainty, considering different levels of input uncertainty. In Section 3.2 realistic uncertainty levels associated to each input parameter are introduced to see which parameters realistically contribute more to the model output uncertainty. Section 3.3 investigates how the variation of input parameters influences the output uncertainty. From this analysis, minimum and maximum limits of output uncertainty are derived within which a spectral simulation based on the same sources of input parameters is supposed to lie. Finally, the main conclusions are summarized in Section 4.

\section{Methodology}

\subsection{SDISORT and the radiative transfer tool UVSPEC}

The propagation of energy in form of electromagnetic radiation from Sun to Earth's surface is affected by phenomena of scattering, absorption and emission caused by the interaction with the Earth's atmosphere. This process is mathematically described by the Radiative Transfer Equation (RTE) (Smith, 1985) that, given a set of input parameters, can be solved in a numerical way with a RTM. One of the most used RTE solvers is the Discrete Ordinates Radiative Transfer Program for a MultiLayered Plane-Parallel Medium (DISORT) (Stamnes et al., 1988). A complete description of DISORT and its implementation can be found in Stamnes et al. (2000). In this study a modified version of DISORT is used to take the sphericity of the Earth's atmosphere into account, namely SDISORT (Dahlback and Stamnes, 1991). Both DISORT and SDISORT are implemented in the UVSPEC tool (Kylling, 1992; Mayer et al., 1997) and included in the libRadtran software package (Mayer and Kylling, 2005). In addition to ten different RTE solvers, UVSPEC provides several options to define the properties of multilayer atmospheric constituents (molecules, aerosol particles, water and ice clouds), and a surface as lower boundary. Several parameters are considered as input to model: surface albedo, extraterrestrial spectrum, solar zenith angle, aerosol properties, water and ozone column. These parameters are widely recognized as those that influence the output most (Manninen et al., 2012; Eltbaakh et al., 2011; Betts, 2005). Each input parameter and its source is introduced below. In general, data time series are collected from available online datasets or tools.

The extraterrestrial spectrum $(S)$ used in this study is derived by Gueymard (2004) from the analysis and synthesis of twenty-three existing measured or modelled spectra.

Solar zenith angle $(\theta)$ is determined with the tool SolPos distributed by the National Renewable Energy Laboratory (NREL, 2015), by giving a specific location and time of the day as input.

Surface albedo $(A)$, i.e. the ratio of reflected radiation from the surface to incident radiation upon it, can be downloaded from CMSAF platform (CMSAF, 2015), which collects data retrieved from the Advanced Very High 
Resolution Radiometer (AVHRR) onboard the polar orbiting NOAA and MetOp satellites. These values are wavelength-independent weekly averages on $15 \times 15 \mathrm{~km}^{2}$ surfaces.

Values of total ozone column $(o)$ are available from the WDC platform (WDC, 2015), level 3 data quality. They are retrieved from GOME-2 instrument mounted onboard the EUMETSATs MetOp-A satellite, and refer to daily averages with a spatial resolution of $80 \times 40 \mathrm{~km}^{2}$.

Total precipitable water (or water vapor, $w$ ) and aerosol properties are obtained from NASA AERONET network (Holben et al., 1998) from values measured with a CIMEL sunphotometer at Kanzelhöhe Observatory station (Austria). This site is in fact taken as a reference for the calculations performed in this study. Values are measured several times per day, and are interpolated in order to get the value corresponding to a specific time of the day. In particular, the aerosol properties used in this study are the Angström exponent $(\alpha)$ and Ångström turbidity coefficient $(\beta)$, single scattering albedo $(\omega)$ and asymmetry factor $(g)$. Angström parameters $\alpha$ and $\beta$ are derived from the Angström law $\delta=\beta \cdot \lambda^{\alpha}$, where $\lambda$ is the wavelength in $\mu \mathrm{m}$ and $\delta$ is the Aerosol Optical Depth (AOD) at that wavelength. The parameter $\alpha$ is related to the distribution of aerosol particle size. In particular, higher values of $\alpha$ indicate a predominance of small particles, while lower values of $\alpha$ indicate a predominance of big particles. The parameter $\beta$ is related to the amount of aerosol particles. In our case, the Angström exponent $\alpha$ is obtained from AERONET AOD measurements between $440 \mathrm{~nm}$ and $870 \mathrm{~nm}$, while the turbidity $\beta$ is obtained from the $\alpha$ value and AOD at $1020 \mathrm{~nm}$. Single scattering albedo $\omega$ represents the ratio of scattering efficiency to total extinction (absorption plus scattering) efficiency. This parameter therefore indicates the probability that the incoming irradiance will be scattered rather than absorbed when interacting with aerosol particles. The parameter $\omega$ is determined as the weighted average of $\omega$ from AERONET in the wavelengths $441,674,870$ and $1020 \mathrm{~nm}$. Finally, aerosol asymmetry factor $g$ indicates states between a complete forward scattering $(g=1)$ and a complete backward scattering $(g=-1)$. It is determined as the value of $g$ from AERONET at $441 \mathrm{~nm}$.

In addition to the nine input parameters described above, the following information and options are set:

- A standard atmosphere referred to as US Standard, 1976 (NASA, 1976). This model describes the idealized, steady-state atmosphere for moderate solar activity by providing singles profiles of temperature, pressure, density, acceleration caused by gravity, pressure scale height, number density, mean particle speed, mean collision frequency, mean free path, mean molecular weight, sound speed, dynamic viscosity, kinematic viscosity, thermal conductivity and geopotential altitude, from 0 to $1000 \mathrm{~km}$. Computations are based on the perfect gas theory and a series of adopted constants, and have been corroborated by rocket and satellite data. The standard atmospheric profile is then scaled according to the values of the different input parameters. No scaling is performed for trace gases since their influence on the spectrum at Earth's surface was found to be negligible.

- Spectral range: from $280 \mathrm{~nm}$ to $2500 \mathrm{~nm}$. This spectral range contains the regions: ultraviolet-B (UV-B, from $280 \mathrm{~nm}$ to $315 \mathrm{~nm}$ ), ultraviolet-A (UV-A, from $315 \mathrm{~nm}$ to $380 \mathrm{~nm}$ ), visible (VIS, from $380 \mathrm{~nm}$ to $780 \mathrm{~nm}$ ), near infrared (NIR, from $780 \mathrm{~nm}$ to $1400 \mathrm{~nm}$ ) and part of short wave infrared (SWIR, from $1400 \mathrm{~nm}$ to $3000 \mathrm{~nm}$ ). The range is broad enough to include the spectral responsivity of all commercially available photovoltaic modules, usually ranging from $300 \mathrm{~nm}$ to $1300 \mathrm{~nm}$ (Silverman et al., 2014), and next generation PV technologies currently in the lab stadium, as well as of spectroradiometers (Galleano et al., 2015).

- An aerosol model, which is then modified according to the aerosol properties provided as input, referred to as aerosol default, and corresponding to the model by Shettle and aerosols (1990): a rural type aerosol in the boundary layer, background aerosol above $2 \mathrm{~km}$, spring-summer conditions and a visibility of $50 \mathrm{~km}$.

- Six streams approximation for the solution of the radiative transfer equation.

A reference set of input parameters is set, corresponding to the atmospheric state at 10:00 (UTC0 time) on April 25th, 2013 at Kanzelhöhe Observatory (N 46.678 , E $13.907^{\circ}$, altitude $1526 \mathrm{~m}$ a.s.1.). This time is chosen since the quality of data measured with a sunphotometer is high for that day (level 2 observations: cloud-screened and quality-assured), and the sky is clear for the whole day. The above mentioned conditions are of course not representative of all the possible atmospheric conditions under clear sky. In any case, the effect of the deviation of input parameters from the reference values on the model output uncertainty is also discussed in Section 3.3.

A first run of UVSPEC program is performed with the set of values shown in Table 1, in order to generate the reference spectrum of global horizontal irradiance (GHI), diffuse horizontal irradiance (DiffHI), and direct horizontal irradiance (DirHI). The spectrally resolved GHI is compared to the measurement taken at the same time

Table 1

Reference set of input parameters for SDISORT model. Values refer to 10:00 (UTC0 time) on April 25th, 2013 at Kanzelhöhe Observatory site.

\begin{tabular}{ll}
\hline Parameter & Reference value \\
\hline extraterrestrial spectrum $(S)$ & Gueymard $(2004)$ \\
solar zenith angle $(\theta)$ & $35.85^{\circ}$ \\
surface albedo $(A)$ & 0.12 \\
ozone column $(o)$ & $321.27 \mathrm{DU}$ \\
water vapor $(w)$ & $7.43 \mathrm{~mm}$ \\
Angström exponent $(\alpha)$ & 1.13 \\
Angström turbidity coefficient $(\beta)$ & 0.025 \\
aerosol single scattering albedo $(\omega)$ & 0.99 \\
aerosol asymmetry factor $(g)$ & 0.67
\end{tabular}


and place with a spectroradiometer EKO MS-710 in the range $350-900 \mathrm{~nm}$, as shown in Fig. 1. The broadband irradiance value (integral value of the spectrum) is $469.9 \mathrm{~W} / \mathrm{m}^{2}$ for measured spectrum and $466.3 \mathrm{~W} / \mathrm{m}^{2}$ for simulated spectrum, while the corresponding Mean Absolute Error (MAE), Mean Bias Error (MBE) and Root Mean Square Error (RMSE) are, respectively, $-6.4 \mathrm{~W} / \mathrm{m}^{2}, 20.1 \mathrm{~W} / \mathrm{m}^{2}$ and $23.7 \mathrm{~W} / \mathrm{m}^{2}$. The absolute values of MAE, MBE, and RMSE, divided by the integral of the measured solar spectrum, give a relative value of $-1.4 \%$ for MAE, of $4.3 \%$ for MBE and of 5.0\% for RMSE. These results are in line with the values of MBE and RMSE calculated from an outdoor intercomparison of ten spectroradiometers from five institutions (Habte et al., 2014), ranging between $-3.5 \%$ and $5.7 \%$ for MBE and between $2.7 \%$ and $7.9 \%$ for RMSE (values determined considering only the spectral range between $380 \mathrm{~nm}$ and $900 \mathrm{~nm}$ ). The instrumental uncertainty of the EKO MS-710 has not been experimentally determined. However, this is the combination of calibration uncertainty and measurement repeatability once the spectroradiometer is positioned outdoor. In particular, the calibration uncertainty is dependent upon the metrology laboratory conditions during calibration, such as the quality of spectral irradiance lamp standards, the relative humidity, the ambient temperature and the geometry of source and optical receiver (Yoon and Gibson, 2011; Myers and Andreas, 2004). A summary of calibration uncertainties of spectroradiometers from different research institutes is given by Galleano et al. (2014).

\subsection{Monte Carlo method for uncertainty propagation}

Given a quantity $Q$, resulting from a measurement process, the uncertainty associated to $Q$ is defined as the dispersion of the value that could be attributed to the measurand (GUM - JCGM 100, 2008). The dispersion of values for $Q$ is described by a Probability Density Function (PDF). If the PDF is known, the standard uncertainty of $Q$ corresponds to its standard deviation. In most cases, the measurand $Q$ is not measured directly, but is determined

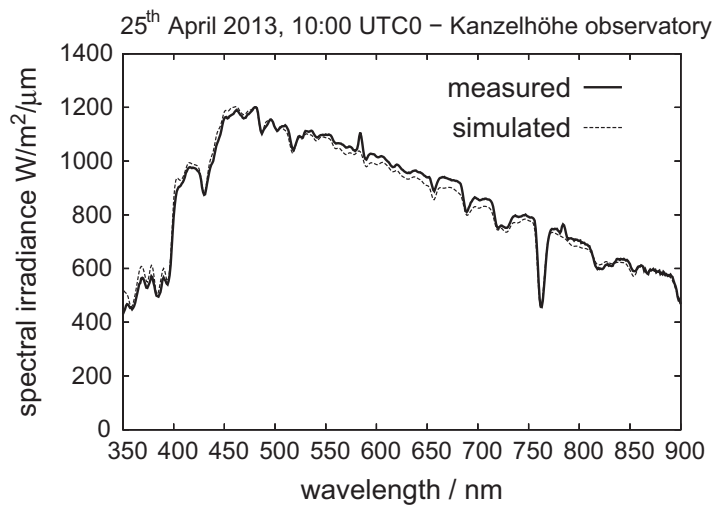

Fig. 1. Comparison of solar spectrum measured with an EKO MS-710 spectroradiometer at 10:00 (UTC0 time) on April 25th, 2013 at Kanzelhöhe Observatory site and the solar spectrum simulated with SDISORT using the reference set of input parameters as in Table 1. through a measurement model, i.e. $Q=M(\mathbf{P})$, where $\mathbf{P}=\left(P_{1}, P_{2}, \ldots, P_{N_{p a r}}\right)$ is a vector of $N_{p a r}$ input quantities that can be themselves measured or dependent from other quantities. The dispersion of the values of such $N_{p a r}$ input quantities is defined by PDFs. If the model $Q=M(\mathbf{P})$ is linear or nonlinear, but differentiable, the standard uncertainty of $Q$ can be obtained from the law of propagation of uncertainty (GUM - JCGM 100, 2008). This turns to be a difficult task when the model is nonlinear and not differentiable. In this case, the Monte Carlo method is more appropriate. This technique consists in approximating the PDF of the quantity $Q$ in a numerical way, by making random draws from the probability distributions of the input quantities, and evaluating the model at the resulting values. In a first step, PDFs are assigned to input quantities $P_{1}, P_{2}, \ldots, P_{N_{p a r}}$. Next, a computer algorithm generates an input vector $p_{1}=\left(p_{1,1}, p_{1,2}, \ldots, p_{1, N_{p a r}}\right)$, where each element $p_{1, j}$ of the vector is calculated according to the PDF of the $j$ th input parameter. The input vector is applied to the model $Q=M(\mathbf{P})$, in order to generate the output value $q_{1}$. The generation of input vectors and corresponding output values is repeated $N$ times, where $N \gg 1$. At the end of the process, a series of $N$ output values is available, the frequency distribution of which corresponds to the PDF of the quantity $Q$. The standard uncertainty of $Q$ is finally determined by calculating the standard deviation of the series of $N$ output values.

The numerically-solved Radiative Transfer Equation implemented in SDISORT can be assimilated to a model $Q=M(\mathbf{P})$ where $\mathbf{P}$ is the vector of input parameters. Being not linear and not differentiable, the Monte Carlo approach can be applied to determine model uncertainty. This requires the knowledge of the PDF of all model input parameters. Unfortunately, SDISORT input values correspond to meteorological and climatological measurements that are not possible to perform directly under repeatability conditions. In other words, it is not possible to assign a PDF to each input parameter simply from measurements. A possible approach to follow is the methodology described by Cordero et al. (2007), that consists in assigning PDFs according to the principle of maximum entropy (GUM - JCGM 101, 2008). This means to select one of the most probable PDFs among those that comply with the restrictions imposed by the available information. In our case for a single measured value $p_{j}$ of the input quantity $P_{j}$, an error bound $d_{j}$ that corresponds to the maximum error reasonably attributed to $p_{j}$ is chosen based on experience or from literature. When only the error bound information is available, the most probable PDF is a rectangular distribution over the interval $\left(p_{j}-d_{j}, p_{j}+d_{j}\right)$ (Cordero et al., 2007), i.e. a distribution that associates the same probability to all value in the considered interval, and the standard uncertainty related to $p_{j}$ is:

$u\left(p_{j}\right)=\frac{d_{j}}{\sqrt{3}}$ 
Once the PDF shape, the error bound and a single measured value are known for each input parameter, it is possible to perform $N \gg 1$ draws, i.e. to generate $N \gg 1$ values of each input parameter. In our case the free software Statistics101 implementing the Monte Carlo algorithm (Grosberg, 2015) is used and a number of $N=500$ draws is found to be sufficient for the analysis. The generated values are combined in $N=500$ input vectors and fed into SDISORT in order to generate $N=500$ outputs of GHI, DiffHI and DirHI spectra. A statistical analysis is therefore performed, consisting in the calculation of the standard deviation $u_{G}$, the mean value $I_{G}$ and the ratio $u_{G} / I_{G}$ of the pool of $N=500$ spectral irradiance values at each wavelength between $280 \mathrm{~nm}$ and $2500 \mathrm{~nm}$. The first of the mentioned quantities corresponds to the standard uncertainty (or absolute uncertainty, expressed in $\mathrm{W} / \mathrm{m}^{2}$ / $\mathrm{nm}$ ), the latter to the relative standard uncertainty (expressed as a percentage). In this work the term relative standard uncertainty is often substituted with the term uncertainty for simplification reasons. The same kind of analysis is performed also on the pool of $N=500$ values of broadband irradiance (corresponding to the integral values of the spectra), and can be theoretically performed on any kind of quantity deriving from spectral irradiance.

The application of the methodology is schematized in Fig. 2. In particular, the described methodology involves simultaneous draws of all input parameters and is used to analyze the combined effect of the input parameters uncertainty on SDISORT output uncertainty. Instead, Fig. 3 represents a similar process that involves draws of an input parameter at a time, while leaving the other input parameters equal to their reference value as in Table 1. This second option is applied to study the effect of one specific input parameter uncertainty on SDISORT output uncertainty.

\section{Simulation results}

\subsection{Impact of input parameters uncertainty on SDISORT uncertainty}

In a first instance, we perform a sensitivity analysis of the uncertainty of SDISORT ascribable to each input quantity: by assuming different values of error bounds for each input parameter, we want to investigate how the

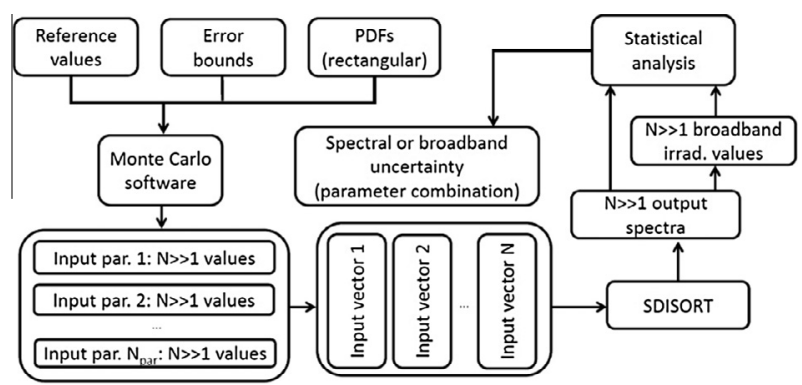

Fig. 2. Schematization of the methodology involving the use of Monte Carlo technique for the evaluation of SDISORT uncertainty given by the simultaneous propagation of the uncertainties of all input parameters.

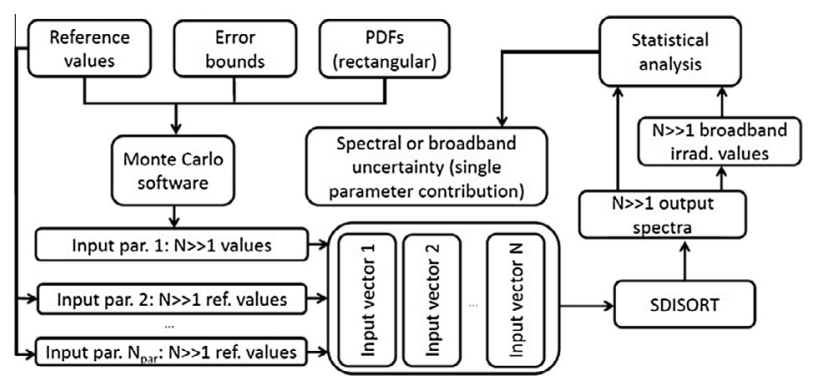

Fig. 3. Schematization of the methodology involving the use of Monte Carlo technique for the evaluation of SDISORT uncertainty given by the propagation of the uncertainty of only one specific input parameter (in the example, input parameter 1).

uncertainty of the model output varies. Therefore, ten different error bounds are assigned, indicated as percentage: $\pm 0.1 \%, \pm 0.2 \%, \pm 0.5 \%, \pm 1 \%, \pm 2 \%, \pm 5 \%, \pm 10 \%, \pm 20 \%$, $\pm 50 \%, \pm 100 \%$. Each percentage corresponds to the maximum variation of input value with respect to the corresponding reference value listed in Table 1 . Note that for the extraterrestrial spectrum the error bound corresponds to the same percent shift at all wavelengths with respect to the reference one. Therefore for this case, by applying the assigned error bounds, only the integral value of the input extraterrestrial spectrum changes, while the shape remains the same.

The methodology described in the previous section and schematized in Fig. 3 is therefore applied. Table 2 shows the values of relative standard uncertainty of broadband irradiance values generated by UVSPEC tool. This is useful to get a hint on the contribution of each input parameter to the uncertainty of the model output, once a specific error bound has been assigned. In particular, the highest values of uncertainty seem to be related to the diffuse component of solar spectrum. It is also interesting to see that the effect of the variability of extraterrestrial spectrum uncertainty propagates in the same way within GHI, DiffHI and DirHI. On the other hand, no indication can be inferred for the combined effect of two or more input parameters from the calculation of the sum of squares of each contribution, since correlations exist and affect the combined uncertainty.

\subsection{Analysis of SDISORT uncertainty contributors}

In the previous section, the relative standard uncertainty of the model output has been evaluated after assigning several levels of error bounds to the different input parameters. In order to deepen our analysis, we now assign a specific and reasonable set of error bounds to the input parameters. We therefore focus on a real case to see which parameters contribute more to the model output uncertainty, and how their effect varies at different spectral regions, as well as on a broadband level. As mentioned in Section 2.2, the value of error bound strictly depends upon the characteristics of the measurement instrument and 
Table 2

Relative standard uncertainty (values in percentage) of SDISORT due to the uncertainty of input parameters, at varying error bound, for each component GHI, DiffHI and DirHI. The results are obtained considering the uncertainty of one input parameter at time applied (as schematized in Fig. 3 ) to the corresponding reference value as in Table 1. n.a.: not affected. $S$ : extraterrestrial spectrum, $\theta$ : solar zenith angle, $A$ : surface albedo, $o$ : ozone column, $w$ : water vapor, $\alpha$ : Ångström exponent, $\beta$ : Ångström turbidity coefficient, $\omega$ : aerosol single scattering albedo, $g$ : aerosol asymmetry factor.

\begin{tabular}{|c|c|c|c|c|c|c|c|c|c|c|}
\hline Parameter & $0.1 \%$ & $0.2 \%$ & $0.5 \%$ & $1 \%$ & $2 \%$ & $5 \%$ & $10 \%$ & $20 \%$ & $50 \%$ & $100 \%$ \\
\hline & \multicolumn{10}{|c|}{ Error bound - global horizontal irradiance } \\
\hline$S$ & 0.06 & 0.12 & 0.29 & 0.58 & 1.11 & 2.95 & 5.61 & 11.60 & 29.17 & 56.69 \\
\hline$\theta$ & 0.03 & 0.06 & 0.15 & 0.29 & 0.56 & 1.47 & 2.81 & 5.77 & 14.64 & 31.75 \\
\hline$A$ & $<0.01$ & $<0.01$ & $<0.01$ & $<0.01$ & 0.01 & 0.03 & 0.05 & 0.10 & 0.25 & 0.51 \\
\hline$o$ & $<0.01$ & $<0.01$ & $<0.01$ & 0.01 & 0.01 & 0.04 & 0.07 & 0.15 & 0.37 & 0.80 \\
\hline$w$ & $<0.01$ & $<0.01$ & 0.01 & 0.02 & 0.04 & 0.09 & 0.18 & 0.37 & 0.96 & 2.47 \\
\hline$\alpha$ & $<0.01$ & $<0.01$ & $<0.01$ & $<0.01$ & $<0.01$ & 0.01 & 0.02 & 0.04 & 0.09 & 0.21 \\
\hline$\beta$ & $<0.01$ & $<0.01$ & $<0.01$ & $<0.01$ & 0.01 & 0.02 & 0.03 & 0.06 & 0.16 & 0.32 \\
\hline$\omega$ & $<0.01$ & 0.01 & 0.01 & 0.03 & 0.05 & 0.13 & 0.24 & 0.46 & 1.04 & 1.95 \\
\hline \multirow[t]{2}{*}{$g$} & $<0.01$ & $<0.01$ & $<0.01$ & 0.01 & 0.01 & 0.04 & 0.07 & 0.15 & 0.36 & 0.60 \\
\hline & \multicolumn{10}{|c|}{ Error bound - diffuse horizontal irradiance } \\
\hline$S$ & 0.06 & 0.12 & 0.29 & 0.58 & 1.11 & 2.95 & 5.61 & 11.60 & 29.17 & 56.69 \\
\hline$\theta$ & 0.01 & 0.02 & 0.05 & 0.09 & 0.18 & 0.47 & 0.89 & 1.84 & 4.88 & 12.03 \\
\hline$A$ & 0.01 & 0.01 & 0.03 & 0.05 & 0.10 & 0.27 & 0.51 & 1.06 & 2.66 & 5.37 \\
\hline$o$ & $<0.01$ & $<0.01$ & 0.01 & 0.02 & 0.03 & 0.08 & 0.15 & 0.32 & 0.82 & 2.06 \\
\hline$w$ & $<0.01$ & $<0.01$ & $<0.01$ & 0.01 & 0.01 & 0.03 & 0.06 & 0.13 & 0.34 & 0.83 \\
\hline$\alpha$ & 0.01 & 0.02 & 0.06 & 0.12 & 0.23 & 0.60 & 1.15 & 2.37 & 6.01 & 12.47 \\
\hline$\beta$ & 0.02 & 0.05 & 0.12 & 0.23 & 0.44 & 1.16 & 2.21 & 4.56 & 11.47 & 22.92 \\
\hline$\omega$ & 0.03 & 0.06 & 0.15 & 0.30 & 0.57 & 1.40 & 2.52 & 4.90 & 11.45 & 21.99 \\
\hline \multirow[t]{2}{*}{$g$} & 0.01 & 0.02 & 0.04 & 0.08 & 0.15 & 0.41 & 0.77 & 1.59 & 3.85 & 6.68 \\
\hline & \multicolumn{10}{|c|}{ Error bound - direct horizontal irradiance } \\
\hline$S$ & 0.06 & 0.12 & 0.29 & 0.58 & 1.11 & 2.95 & 5.61 & 11.60 & 29.17 & 56.69 \\
\hline$\theta$ & 0.03 & 0.06 & 0.16 & 0.31 & 0.60 & 1.58 & 3.01 & 6.17 & 15.66 & 33.92 \\
\hline$A$ & n.a. & n.a. & n.a. & n.a. & n.a. & n.a. & n.a. & n.a. & n.a. & n.a. \\
\hline$o$ & $<0.01$ & $<0.01$ & $<0.01$ & 0.01 & 0.01 & 0.03 & 0.06 & 0.13 & 0.32 & 0.68 \\
\hline$w$ & $<0.01$ & $<0.01$ & 0.01 & 0.02 & 0.04 & 0.10 & 0.19 & 0.40 & 1.03 & 2.64 \\
\hline$\alpha$ & $<0.01$ & $<0.01$ & 0.01 & 0.01 & 0.03 & 0.07 & 0.14 & 0.29 & 0.73 & 1.58 \\
\hline$\beta$ & $<0.01$ & 0.01 & 0.01 & 0.03 & 0.05 & 0.14 & 0.26 & 0.54 & 1.36 & 2.77 \\
\hline$\omega$ & n.a. & n.a. & n.a. & n.a. & n.a. & n.a. & n.a. & n.a. & n.a. & n.a. \\
\hline$g$ & n.a. & n.a. & n.a. & n.a. & n.a. & n.a. & n.a. & n.a. & n.a. & n.a. \\
\hline
\end{tabular}

method, and can be derived either from previous studies or from experience. In our case we apply a security factor to values of uncertainty available in the literature, provided that the data type, the measurement instruments and the boundary conditions (for example, some values of uncertainty are valid at specific ranges of solar zenith angle or are associated to other specific atmospheric conditions) are ascribable to our case. The set of selected error bounds and the literature source for input uncertainties are reported in Table 3.

As before, for each input parameter a series of $N=500$ values lying within the error bounds specified in Table 3 is generated according to a uniform distribution with a Monte Carlo based computer software. In a first step, the generated values are fed into SDISORT to generate just as many GHI, DiffHI and DirHI spectra, in such a way that only one input parameter at a time varied, while assigning reference values to the remaining ones (methodology as in Fig. 3). Later, all generated values are fed into SDISORT simultaneously, in order to account for the simultaneous and interacting effect of the uncertainty of input parameters (methodology as in Fig. 2). Results are shown in Fig. 4. Plots on the left show the whole spectral range $280-2500 \mathrm{~nm}$ and is useful to identify the major contributors in the VIS, NIR and SWIR regions, while plots on the right are scaled in order to focus on the UV-B and UV-A parts. The values of output uncertainty of broadband GHI, DiffHI and DirHI, for each considered spectral region and for the whole range, are reported in Table 4. First of all, it is verified that the combined uncertainty does not correspond to the sum of squares of each contributor. This is expected, since some correlation exist between input parameters within the radiative transfer equation, and confirms the suitability of a Monte Carlo based approach for this kind of analysis.

The major contributors of SDISORT output uncertainty for GHI, given the set of error bounds shown in Table 3, are extraterrestrial spectrum and Angström turbidity coefficient $\beta$. The effect of extraterrestrial spectrum on uncertainty propagation is constant for the whole spectral range, around $2.95 \%$. The influence of Angström turbidity coefficient $\beta$ is more evident in the UV region (broadband uncertainty of $0.84 \%$ at UV-B and $0.65 \%$ at UV-A, with a local spectral uncertainty peak of around $1.4 \%$ ), while it decreases at increasing wavelengths, with 
Table 3

Error bounds associated to the input parameters measured at 10:00 on April 25th, 2013 at Kanzelhöhe Observatory site, and listed in Table 1.

\begin{tabular}{lll}
\hline Parameter & Error bound & Source \\
\hline$S$ & $5 \%$ & Gueymard (2004) \\
$\theta$ & $0.03^{\circ}$ & NREL (2015) \\
$A$ & $25 \%$ & Xia et al. (2007) \\
$o$ & $5 \%$ & Valks et al. (2011) \\
$w$ & $10 \%$ & Holben et al. (2001), Perez-Ramirez et al. (2014) \\
$\alpha$ & 0.08 & Schuster et al. (2006), Toledano et al. (2007) \\
$\beta$ & 0.025 & Holben et al. (1998), Eck et al. (2001) \\
$\omega$ & 0.05 & Dubovik et al. (2000) \\
$g$ & 0.05 & Xia et al. (2007), Andrews et al. (2006) \\
\hline
\end{tabular}
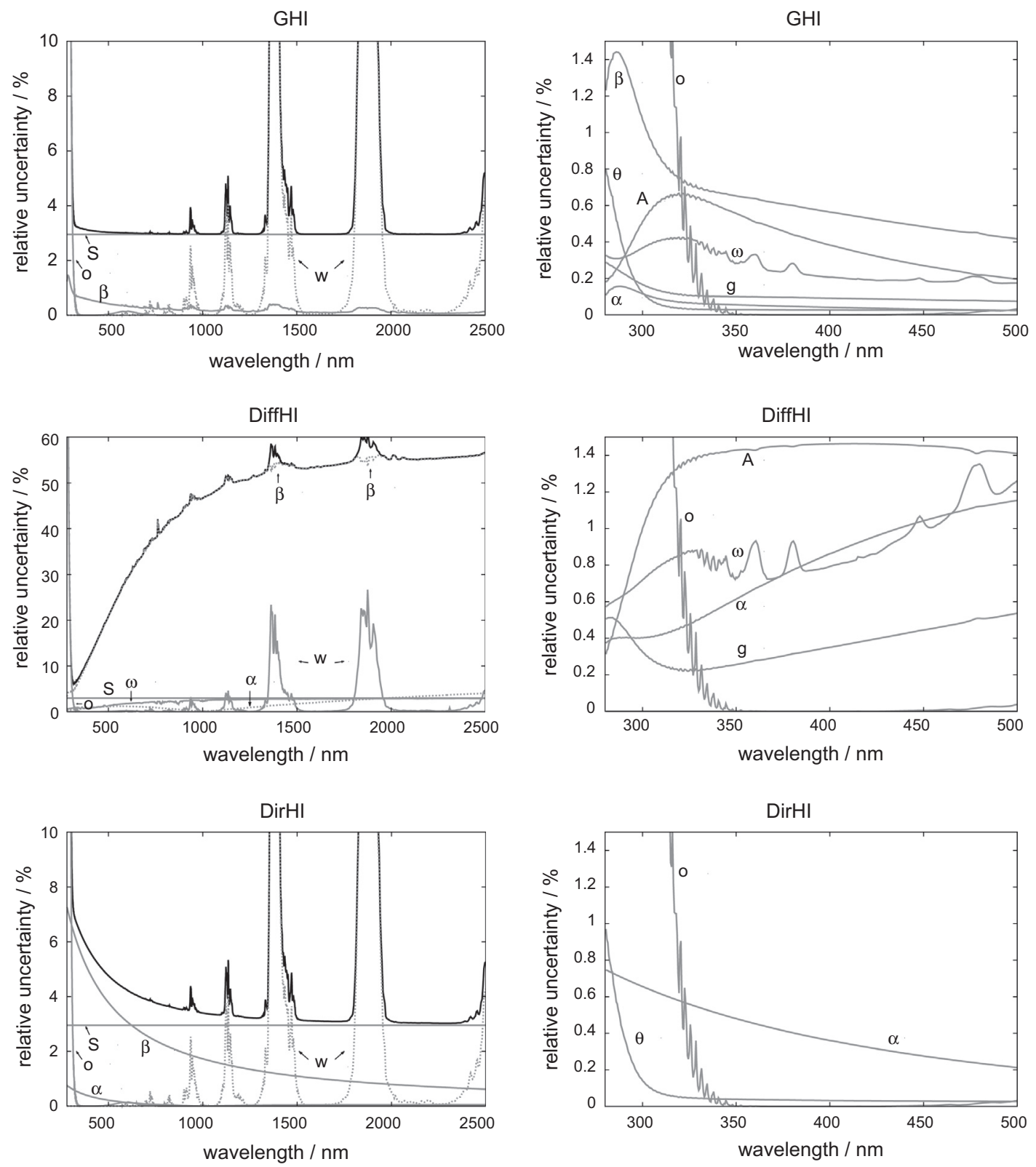

Fig. 4. Relative uncertainty (values in percentage) of the SDISORT output at varying wavelength, due to the uncertainty of each input parameter and to the combined effect of all input parameters, for GHI, DiffHI and DirHI. (left) view of the whole investigated spectral range (uncertainty due to combined effect is in black). (right) zoom on the UV part of the spectrum. $S$ : extraterrestrial spectrum, $\theta$ : solar zenith angle, $A$ : surface albedo, $o$ : ozone column, $w$ : water vapor, $\alpha$ : Angström exponent, $\beta$ : Ångström turbidity coefficient, $\omega$ : aerosol single scattering albedo, $g$ : aerosol asymmetry factor. 
Table 4

Uncertainty of SDISORT output ascribable to the uncertainty of the different input parameters and to their simultaneous effect (in italics) computed on the broadband values of GHI, DiffHI and DirHI in several spectral regions, and in the whole considered spectral range. n.a.: not affected. $S$ : extraterrestrial spectrum, $\theta$ : solar zenith angle, $A$ : surface albedo, $o$ : ozone column, $w$ : water vapor, $\alpha$ : Angström exponent, $\beta$ : Angström turbidity coefficient, $\omega$ : aerosol single scattering albedo, $g$ : aerosol asymmetry factor.

\begin{tabular}{|c|c|c|c|c|c|c|c|c|c|c|}
\hline Spectral range & $S$ & $\theta$ & $A$ & $o$ & $w$ & $\alpha$ & $\beta$ & $\omega$ & $g$ & Simultaneous effects \\
\hline & \multicolumn{10}{|c|}{ Global horizontal irradiance } \\
\hline UV-B (280-315 nm) & 2.95 & 0.05 & 0.63 & 3.02 & $<0.01$ & 0.08 & 0.84 & 0.41 & 0.13 & 4.42 \\
\hline UV-A (315-380 nm) & 2.95 & 0.03 & 0.55 & 0.09 & $<0.01$ & 0.05 & 0.65 & 0.31 & 0.10 & 3.13 \\
\hline VIS $(380-780 \mathrm{~nm})$ & 2.95 & 0.02 & 0.16 & 0.05 & 0.03 & 0.02 & 0.38 & 0.16 & 0.07 & 3.00 \\
\hline NIR (780-1400 nm) & 2.95 & 0.02 & 0.03 & $<0.01$ & 0.34 & 0.00 & 0.20 & 0.08 & 0.04 & 2.98 \\
\hline SWIR $(1400-2500 \mathrm{~nm})$ & 2.95 & 0.03 & 0.01 & $<0.01$ & 0.62 & 0.01 & 0.11 & 0.04 & 0.02 & 3.02 \\
\hline \multirow[t]{2}{*}{ all spectrum $(280-2500 \mathrm{~nm})$} & 2.95 & 0.02 & 0.12 & 0.04 & 0.18 & 0.01 & 0.31 & 0.13 & 0.06 & 2.99 \\
\hline & \multicolumn{10}{|c|}{ Diffuse horizontal irradiance } \\
\hline UV-B $(280-315 \mathrm{~nm})$ & 2.95 & 0.04 & 1.24 & 3.17 & $<0.01$ & 0.42 & 4.61 & 0.81 & 0.25 & 6.69 \\
\hline UV-A (315-380 nm) & 2.95 & 0.01 & 1.42 & 0.12 & $<0.01$ & 0.61 & 7.28 & 0.81 & 0.26 & 8.19 \\
\hline VIS $(380-780 \mathrm{~nm})$ & 2.95 & 0.01 & 1.35 & 0.05 & 0.02 & 1.06 & 21.81 & 1.34 & 0.57 & 22.11 \\
\hline NIR (780-1400 nm) & 2.95 & 0.01 & 0.82 & $<0.01$ & 0.35 & 0.13 & 45.28 & 2.35 & 1.16 & 44.83 \\
\hline SWIR (1400-2500 nm) & 2.95 & 0.01 & 0.56 & $<0.01$ & 0.68 & 2.35 & 54.05 & 2.81 & 1.47 & 53.19 \\
\hline \multirow[t]{2}{*}{ all spectrum $(280-2500 \mathrm{~nm})$} & 2.95 & 0.01 & 1.29 & 0.08 & 0.06 & 0.82 & 22.20 & 1.37 & 0.59 & 22.47 \\
\hline & \multicolumn{10}{|c|}{ Direct horizontal irradiance } \\
\hline UV-B (280-315 nm) & 2.95 & 0.07 & n.a. & 2.86 & $<0.01$ & 0.61 & 6.46 & n.a. & n.a. & 7.80 \\
\hline UV-A (315-380 nm) & 2.95 & 0.04 & n.a. & 0.07 & $<0.01$ & 0.47 & 5.60 & n.a. & n.a. & 6.42 \\
\hline VIS $(380-780 \mathrm{~nm})$ & 2.95 & 0.03 & n.a. & 0.05 & 0.03 & 0.16 & 3.35 & n.a. & n.a. & 4.51 \\
\hline NIR (780-1400 nm) & 2.95 & 0.02 & n.a. & $<0.01$ & 0.34 & 0.00 & 1.76 & n.a. & n.a. & 3.46 \\
\hline SWIR (1400-2500 nm) & 2.95 & 0.03 & n.a. & $<0.01$ & 0.62 & 0.04 & 0.92 & n.a. & n.a. & 3.15 \\
\hline all spectrum $(280-2500 \mathrm{~nm})$ & 2.95 & 0.03 & n.a. & 0.03 & 0.20 & 0.10 & 2.62 & n.a. & n.a. & 3.98 \\
\hline
\end{tabular}

local peaks at water absorption bands. Focusing on the short-wavelength region, the influence of ozone on uncertainty propagation affects only the UV-B region $(3.02 \%$ broadband uncertainty), while it can vary locally from around $110 \%$ to $4 \%$. Nevertheless, the effect of the uncertainty of this atmospheric gas on the whole spectral range is negligible. Water vapor is an important source of uncertainty at water absorption bands, especially in the range $1350-1425 \mathrm{~nm}$ and $1800-1950 \mathrm{~nm}$, where the value of spectral uncertainty can be higher than $10 \%$.

The relative uncertainty of DirHI presents similarities with that of GHI, with the only exceptions of Angström turbidity coefficient $\beta$ and Ångström exponent $\alpha$. The first is responsible for a broadband uncertainty of $6 \%$ in the UV region, which decreases but still remain considerable also in the VIS, NIR and SWIR regions with, respectively, $3.35 \%, 1.76 \%$ and $0.92 \%$ broadband uncertainty. The second has a lower influence than $\beta$, with more effects on the UV region of the spectrum.

The simultaneous uncertainty propagation of input parameters to the model output in DiffHI presents different characteristics for wavelengths higher than $315 \mathrm{~nm}$ than the previous cases, with increasing values reaching local peaks of spectral uncertainty of around $60 \%$ in the water absorption region $1800-1950 \mathrm{~nm}$. On the whole spectral range, the uncertainty of broadband DiffHI is $22.37 \%$. This behavior is almost completely attributable to Angström turbidity coefficient $\beta$. The reason for these high values of uncertainty due to $\beta$ is mostly explained by the high uncertainty associated to the AERONET source $(100 \%$, being the reference value equal to 0.025 and the associated error bound equal to 0.025), which derives by the temperaturesensitivity of CIMEL sensor at wavelength $1020 \mathrm{~nm}$ where AOD is measured and from which $\beta$ is derived. In the Version 3 of AERONET database, which will be released in 2016 (Eck, 2015), some corrections will be applied in order to reduce the effects of extreme temperature on AOD (and therefore $\beta$ ) accuracy, thus improving the associated uncertainty (Holben, 2013). The influence of the uncertainty of parameters representing aerosol properties is in general higher for DiffHI than for other cases, and is therefore not negligible: Angström exponent $\alpha$ effect is higher in SWIR region ( $2.35 \%$ broadband uncertainty), while the effect of single scattering albedo and asymmetry factor is higher in NIR and SWIR regions (broadband uncertainties around $2.6 \%$ for $\omega$ and around $1.3 \%$ for $g$ ). The latter presents local peaks of spectral uncertainty at water absorption bands $1350-1425 \mathrm{~nm}$ and $1800-1950 \mathrm{~nm}$ (not visible in Fig. 4, for clarity reasons).

It is important to underline that this analysis is performed at specific values of input parameters referring to reference conditions of Table 1, and uncertainty values cannot therefore be generalized. However, the effect of deviation of input parameters from the reference set will be investigated in Section 3.3 in order to define a validity area within a minimum and maximum uncertainty limits, representing all possible combinations of input parameters.

\subsection{Influence of variability of input parameters}

In this section we analyze the effect of deviation of input parameters from the reference set on SDISORT output, 
supposing that the set of error bounds remain the same as in Table 3. This way, it is possible to define an uncertainty area delimited by a minimum and maximum uncertainty boundaries, and satisfying all possible combinations of input parameters. Different values are assigned to each input parameter based on a specific step and within defined physical limits, and simulations are run in which one input parameter at a time varies according to the error bounds assigned in Table 3, while assigning reference values to the remaining ones (methodology as in Fig. 3). Results are summarized only for broadband GHI in Table 5 that shows, in addition to the selected steps and variability ranges used for the simulations, the values of input parameters that correspond respectively to the minimum and to the maximum output calculated uncertainty, and the values of uncertainties themselves. For example, values within the physical range from $0 \mathrm{~nm}$ to $50 \mathrm{~mm}$ are assigned to water vapor with a $5 \mathrm{~mm}$ step. The associated minimum output uncertainty is at $0 \mathrm{~nm}$, while the maximum output uncertainty is at $50 \mathrm{~nm}$ and corresponds to $0.32 \%$. In the case of extraterrestrial spectrum, no variability range and no step can be assigned since it is not possible to let a reference spectrum (in this case, the one by Gueymard (2004)) vary from a minimum to a maximum physical value. For this case, only an uncertainty value of $2.95 \%$ is assigned, as calculated in Section 3.2 and corresponding to an error bound of $5 \%$.

In general the uncertainty of the model output depends on the value of the input parameter. When error bounds are defined as percentage, the output uncertainty tends to increase at increasing values of input parameter. The only exception is represented by surface albedo, which shows a peak of uncertainty at values around 0.75 . As for error bounds defined as absolute value, the uncertainty of the model output can either increase (as in the case of solar zenith angle, Ångström exponent and Ångström turbidity coefficient), or decrease (as in the case of aerosol single scattering albedo and aerosol asymmetry factor) at increasing values of input parameters. Surface albedo and total precipitable water parameter variation affect the output uncertainty most, with a total variation of output uncertainty of, respectively, $0.86 \%$ and $0.32 \%$. On the other hand, solar zenith angle does not seem to significantly affect SDISORT output uncertainty. The latter result confirms the outcome of the work by Cordero et al. (2007) and extends it to wavelengths higher than $400 \mathrm{~nm}$.

In addition, under the same set of error bounds as in Table 3, two additional simulations are run:

- one with a set of input parameters corresponding to the lowest output uncertainties of broadband GHI (column 3 of Table 5);

- one other with a set of input parameter values corresponding to the highest output uncertainties of broadband GHI (column 4 of Table 5).

This way, it is possible to identify a sort of uncertainty area within which all possible combinations of input parameters with error bounds as in Table 3 are likely to lie. This is reported in Fig. 5 (left), together with the spectral uncertainty due to simultaneous effect of reference input parameters, calculated in Section 3.2. Fig. 5 (right) shows the corresponding values of broadband uncertainty for GHI, for each spectral region. A high variability of SDISORT uncertainty occurs in the UV-B, UV-A and VIS regions, while the maximum and minimum output broadband uncertainties are comparable in the NIR and SWIR regions.

SDISORT uncertainty of broadband GHI in the investigated range $280-2500 \mathrm{~nm}$ ranges between a minimum of $2.9 \%$ and a maximum of $5.9 \%$. It is interesting to notice that the latter value is higher that the value obtained from a simple sum of squares of the single uncertainties ascribable to each input parameter (equal to $3.1 \%$ ), thus confirming once more the suitability of Monte Carlo to such a kind of analysis.

The obtained range of uncertainty can be compared with the uncertainty of spectra measured with spectroradiometers. As mentioned in Section 2.1, the latter is a combination of instrument calibration uncertainty and of repeatability of measurements performed outdoor. For example, Vasiliki et al. (2013) calculate a combined standard uncertainty of $2.5 \%(k=1)$ for outdoor spectral measurements of the global normal irradiance (GNI). This value is lower but still comparable with the uncertainty calculated in this study. Furthermore, the gradual improve-

Table 5

Series of input parameters values selected to run UVSPEC tool, and values corresponding to maximum and minimum output broadband uncertainty for broadband GHI, respectively. Corresponding output uncertainty is displayed into brackets.

\begin{tabular}{llll}
\hline Parameter & Min-max value, step & Min value (unc.) & Max value (unc.) \\
\hline$S$ & Gueymard (2004) & $-(2.95 \%)$ & $-(2.95 \%)$ \\
$\theta$ & $10^{\circ}-70^{\circ}, 10^{\circ}$ & $10^{\circ}(0.01 \%)$ & $70^{\circ}(0.10 \%)$ \\
$A$ & $0.05-0.95,0.10$ & $0.05(0.05 \%)$ & $0.75(0.91 \%)$ \\
$o$ & $250-500 \mathrm{DU}, 25 \mathrm{DU}$ & $250 \mathrm{DU}(0.03 \%)$ & $500 \mathrm{DU}(0.05 \%)$ \\
$w$ & $0-50 \mathrm{~mm}, 5 \mathrm{~mm}$ & $0(0.00 \%)$ & $50(0.32 \%)$ \\
$\alpha$ & $0.5-2.5,0.25$ & $0.5(0.01 \%)$ & $2.5(0.04 \%)$ \\
$\beta$ & $0-0.5,0.05$ & $0(0.16 \%)$ & $0.5(0.36 \%)$ \\
$\omega$ & $0.6-1,0.05$ & $1(0.12 \%)$ & $0.6(0.14 \%)$ \\
$g$ & $0.5-0.9,0.05$ & $0.9(0.04 \%)$ & $0.5(0.06 \%)$ \\
\hline
\end{tabular}



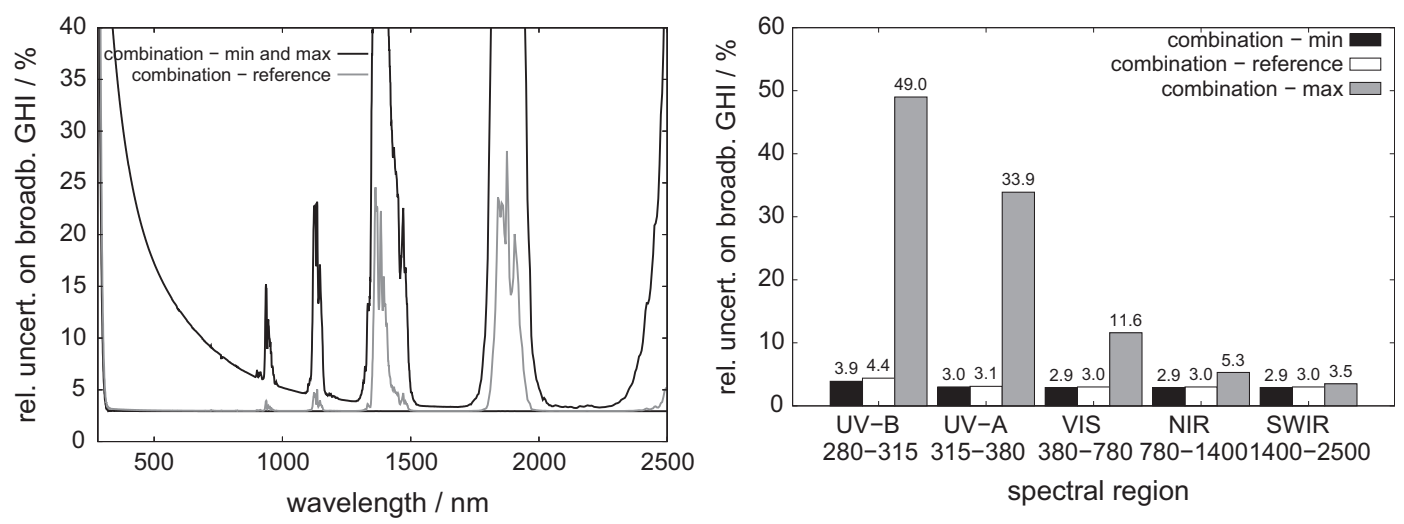

Fig. 5. Max and min relative spectral uncertainty of the SDISORT outputs for GHI due to the combination of input parameters generating, respectively, the highest and lowest uncertainty, and with values of error bounds as defined in Table 3. (left) output spectral uncertainties (right) output broadband uncertainties. Output spectral uncertainty from the reference set of input parameters as in Table 1 is also displayed.

ment of instruments and procedures for the retrieval of RTM input parameters looks promising and will push towards a reduction of uncertainty levels.

\section{Conclusions}

An analysis of uncertainty of SDISORT radiative transfer model implemented in the UVSPEC tool is presented in this paper. A Monte Carlo technique is used to investigate the propagation of the uncertainty of nine different input parameters into the model output. The uncertainty contribution of every single input parameter as well as of their combination to the uncertainty of spectral and broadband modelled irradiance is evaluated. The analysis is performed on the global horizontal irradiance (GHI), the diffuse horizontal irradiance (DiffHI), and the direct horizontal irradiance (DirHI). Results show that DiffHI is generally more affected by uncertainty than DirHI and GHI, both on the spectral and broadband level.

Considering a reference set of input parameters representing the atmospheric state at Kanzelhöhe Observatory (Austria) site at 10:00 on April 25th, 2013, and a set of associated error bounds, it is found that Angström turbidity coefficient $\beta$ explains most of the uncertainty in the broadband DiffHI $(22.47 \%$ in the range $280-2500 \mathrm{~nm}$ ), with increasing values at increasing wavelength. The effect of the uncertainty of this parameter is also evident in the broadband DirHI. The prominent role of $\beta$ is mostly explained by the high value of error bound associated to AERONET source $(100 \%$, being the reference value equal to 0.025 and the associated error bound equal to 0.025 ). The uncertainty of this parameter must be therefore properly taken into consideration especially when dealing with DiffHI. On the other hand, in a new version of AERONET database that will be soon released (Eck, 2015) some corrections should be applied in order to reduce the associated uncertainty of this parameter.

As for the other input parameters, ozone column is an important source of uncertainty for all irradiance components at UV-B regions ( $280-315 \mathrm{~nm})$, with values of output spectral uncertainty of around 3\%, and local peaks reaching also $110 \%$. Extraterrestrial spectrum contributes for $2.95 \%$ of output uncertainty on the whole spectral range. However, efforts done in the last years in synthesizing less uncertain extraterrestrial spectra look promising (Gueymard, 2004). Minor contributions come from Ångström exponent $\alpha$, evident in the SWIR region of DiffHI $(2.35 \%$ broadband uncertainty) and aerosol single scattering albedo in the NIR and SWIR regions of DiffHI $(2.35 \%$ and $2.81 \%$ broadband uncertainty, respectively). Finally, water vapor seems not to contribute significantly to the broadband output uncertainty (less than $0.70 \%$ on all spectral regions and for all broadband irradiance components), but can result in local peaks higher than $10 \%$ at water absorption bands, especially between $1350-1425 \mathrm{~nm}$ and $1800-1950 \mathrm{~nm}$.

By studying the effect of deviation of input parameters from the above mentioned reference set, it is possible to conclude that the maximum uncertainty of broadband GHI calculated with SDISORT in the spectral regions included in the range $280-2500 \mathrm{~nm}$ is significantly high in UV-B, UV-A and VIS regions $(49.0 \%, 33.9 \%$ and $11.6 \%$ respectively), while it tends to be closer to the corresponding minimum levels at NIR and SWIR $(5.3 \%$ and 3.5\% respectively). On the whole considered spectral range $280-2500 \mathrm{~nm}$, the broadband uncertainty of GHI can vary between a minimum of $2.9 \%$ and a maximum of $5.9 \%$. This range is higher, but still comparable to typical uncertainty values of global irradiance measurements performed with spectroradiometers. However, the gradual improvement of instruments and methodology for the derivation of RTM input quantities is bound to decrease the associated levels of uncertainty. The gradual reduction in model uncertainties must go in parallel with a reduction of uncertainty in spectral irradiance calibration and measurement. In fact, a low value of uncertainty of simulated spectrum is useful and valuable only when the model is used to simulate a measured spectrum that is close to the true value. Considering a model uncertainty of around $3-6 \%$ as found by this study, and a current state of the art spectral irradiance measurement uncertainty of $3-5 \%$, combined values 
of $6-11 \%$ actually represent a real estimate of the state of the art limit of error uncertainty in terrestrial solar spectral radiation estimates.

In general this study proves that, provided that the uncertainty of every single input parameter is known, the derivation of the output combined uncertainty with a classical propagation of error always underestimates the more realistic values obtained with a more appropriate technique like Monte Carlo, taking the correlation of input parameters into account.

\section{Acknowledgements}

The authors wish to acknowledge the European Regional Development Fund (ERDF) and the Stiftung Südtiroler Sparkasse for co-financing the Project 2-1a-97 "PV Initiative" and the Project 5-1a-232 "Flexi-BIPV". The author David Moser wishes to acknowledge the European Union's Horizon 2020 research and innovation programme under Grant Agreement No. 649997, Project "Solar Bankability". The author Giorgio Belluardo would like to thank R. Galleano for the fruitful discussion on spectroradiometers uncertainty and T. Eck for the helpful suggestions concerning AERONET data.

\section{References}

Andrews, E., Sheridan, P.J., Fiebig, M., McComiskey, A., Ogren, J.A., Arnott, P., Covert, D., Elleman, R., Gasparini, R., Collins, D., Jonsson, H., Schmid, B., Wang, J., 2006. Comparison of methods for deriving aerosol asymmetry parameter. J. Geophys. Res. Atmos. 111, D05S04.

Betts, T.R., 2005. Investigation of photovoltaic device operation under varying spectral conditions Ph.D. Thesis. Loughborough University.

Clough, S.A., Shephard, M.W., Mlawer, E.J., Delamere, J.S., Iacono, M. J., Cady-Pereira, K., Boukabara, S., Brown, P.D., 2005. Atmospheric radiative transfer modeling: a summary of the AER codes. J. Quant. Spectrosc. Radiat. Transfer 91 (2), 233-244.

CMSAF, 2015. Surface radiation products https://wui.cmsaf.eu/safira/ action/viewProduktList?id=2 (accessed: 2015-05-25).

Cordero, R., Seckmeyer, G., Pissulla, D., Dasilva, L., Labbe, F., 2007. Uncertainty evaluation of the spectral UV irradiance evaluated by using the UVSPEC radiative transfer model. Opt. Commun. 276, 44-53.

Dahlback, A., Stamnes, K., 1991. A new spherical model for computing the radiation field available for photolysis and heating at twilight. Planet. Space Sci. 39, 671-683.

Ding, S., Yang, P., Weng, F., Liu, Q., Han, Y., van Delst, P., Li, J., Baum, B., 2011. Validation of the community radiative transfer model. J. Quant. Spectrosc. Radiat. Transfer 112, 1050-1064.

Dubovik, O., Smirnov, A., Holben, B.N., King, M.D., Kaufman, Y.J., Eck, T.F., Slutsker, I., 2000. Accuracy assessments of aerosol optical properties retrieved from Aerosol Robotic Network (AERONET) Sun and sky radiance measurements. J. Geophys. Res. Atmos. 105, 97919806.

Eck, T., 2015. Private communication.

Eck, T.F., Holben, B.N., Dubovik, O., Smirnov, A., Slutsker, I., Lobert, J.M., Ramanathan, V., 2001. Column-integrated aerosol optical properties over the Maldives during the northeast monsoon for 1998-2000. J. Geophys. Res. Atmos. 106, 28555-28566.

Eltbaakh, Y.A., Ruslan, M.H., Alghoul, M.A., Othman, M.Y., Zaharim, A., Sopian, K., 2011. Effect of cloudless sky parameters on global spectral solar radiation within $0.3-1.1 \mu \mathrm{m}$ region. Proc. 4th WSEAS
International Conference on Energy and Development, Environment and Biomedicine, pp. 209-213.

Galleano, R., Zaaiman, W., Strati, C., Bartocci, S., Pravettoni, M., Marzoli, M., Fucci, R., Leanza, G., Timó, G., Minuto, A., Catena, M., Aleo, F., Takagi, S., Akiyama, A., Nuez, R., Belluardo, G., 2015. Second international spectroradiometer intercomparison: results and impact on PV device calibration. Prog. Photovolt.: Res. Appl. 23 (7), 929-938.

Galleano, R., Zaaiman, W., Virtuani, A., Pavanello, D., Morabito, P., Minuto, A., Spena, A., Bartocci, S., Fucci, R., Leanza, G., Fasanaro, D., Catena, M., 2014. Intercomparison campaign of spectroradiometers for a correct estimation of solar spectral irradiance: results and potential impact on photovoltaic devices calibration. Prog. Photovolt: Res. Appl. 22 (11), 1128-1137.

Grosberg, J., 2015. Statistics 101 [computer software] http://www.statistics101.net/ (accessed: 2015-05-26).

Gueymard, C., 1995. Simple model of the atmospheric radiative transfer of sunshine, version 2 (SMARTS2): algorithms description and performance assessment. Tech. Rep. FSEC-PF-270-95, Florida Solar Energy Center.

Gueymard, C.A., 2004. The sun's total and spectral irradiance for solar energy applications and solar radiation models. Sol. Energy 76 (4), 423-453.

GUM - JCGM 100:2008, 2008. Evaluation of measurement data - guide to the expression of uncertainty in measurement.

GUM - JCGM 101:2008, 2008. Evaluation of measurement data: supplement 1 to the guide to the expression of uncertainty in measurement: propagation of distributions using a Monte Carlo method.

Habte, A., Andreas, A., Ottoson, L., Gueymard, C., Fedor, G., Fowler, S., Peterson, J., Naranen, E., Kobashi, T., Akiyama, A., Takagi, S., 2014. Indoor and outdoor spectroradiometer intercomparison for spectral irradiance measurement Tech. Rep. NREL/TP-5D00-61476. National Renewable Energy Laboratory.

Holben, B., 2013. AERONET update: development of version 3 http:// aeronet.gsfc.nasa.gov/new_web/Publications/quarterlies/Update_Mar7_ Version_3.pdf (accessed: 2015-10-01).

Holben, B., Tanr, D., Smirnov, A., Eck, T.F., Slutsker, I., Abuhassan, N., Newcomb, W., Schafer, J.S., Chatenet, B., Lavenu, F., Kaufman, Y.J., Vande Castle, J., Setzer, A., Markham, B., Clark, D., Frouin, R., Halthore, R., Karneli, A., O’Neill, N., Pietras, C., Pinker, R., Voss, K., Zibordi, G., 2001. An emerging ground-based aerosol climatology: aerosol optical depth from AERONET. J. Geophys. Res. Atmos. 106, 12067-12097.

Holben, B.N., Eck, T.F., Slutsker, I., Tanr, D., Buis, J.P., Setzer, A., Vermote, E., Reagan, J.A., Kaufman, Y.J., Nakajima, T., Lavenu, F., Jankowiak, I., Smirnov, A., 1998. AERONET - a federated instrument network and data archive for aerosol characterization. Remote Sens. Environ. 66 (1), 1-16.

Kylling, A., 1992. Radiation transport in cloudy and aerosol loaded atmospheres Ph.D. Thesis. Alaska University.

Manninen, T., Riihel, A., de Leeuw, G., 2012. Atmospheric effect on the ground-based measurements of broadband surface albedo. Atmos. Meas. Tech. 5 (11), 2675-2688.

Mayer, B., Kylling, A., 2005. Technical note: the libRadtran software package for radiative transfer calculations - description and examples of use. Atmos. Chem. Phys. 5, 1855-1877.

Mayer, B., Seckmeyer, G., Kylling, A., 1997. Systematic long-term comparison of spectral UV measurements and UVSPEC modeling results. J. Geophys. Res. Atmos. 102, 8755-8767.

Myers, D., Andreas, A., 2004. Sensitivity of spectroradiometric calibrations in the near infrared to variations in atmospheric water vapor. Proc. American Solar Energy Society Annual Conference, Portland, Oregon.

Myers, D.R., 2005. Solar radiation modeling and measurements for renewable energy applications: data and model quality. Energy 30 (9), 1517-1531. 
NASA, 1976. U.S. Standard Atmosphere, 1976. Tech. rep., NASA TM-X74335.

NREL, 2015. Solar position and intensity calculator (SOLPOS) http:// www.nrel.gov/midc/solpos/solpos.html (accessed: 2015-05-22).

Perez-Ramirez, D., Whiteman, D.N., Smirnov, A., Lyamani, H., Holben, B.N., Pinker, R., Andrade, M., Alados-Arboledas, L., 2014. Evaluation of AERONET precipitable water vapor versus microwave radiometry, GPS, and radiosondes at ARM sites. J. Geophys. Res. Atmos. 119 (15), 2014JD021730.

Román, R., Bilbao, J., de Miguel, A., 2014a. Solar radiation simulations in the Iberian Peninsula: accuracy and sensitivity to uncertainties in inputs of a radiative transfer model. J. Quant. Spectrosc. Radiat. Transfer 145, 95-109.

Román, R., Bilbao, J., de Miguel, A., 2014b. Uncertainty and variability in satellite-based water vapor column, aerosol optical depth and Ångström exponent, and its effect on radiative transfer simulations in the Iberian Peninsula. Atmos. Environ. 89, 556-569.

Schuster, G.L., Dubovik, O., Holben, B.N., 2006. Angström exponent and bimodal aerosol size distributions. J. Geophys. Res. Atmos. 111, D07207.

Seidel, F.C., Kokhanovsky, A.A., Schaepman, M.E., 2010. Fast and simple model for atmospheric radiative transfer. Atmos. Meas. Tech. 3 (4), 1129-1141.

Shettle, E., aerosols, Models of, 1990. clouds and precipitation for atmospheric propagation studies. In: Proc. AGARD Conference, vol. 454.

Silverman, T., Jahn, U., Friesen, G., Pravettoni, M., Apolloni, M., Louwen, A., van Sark, W., Schweiger, M., Belluardo, G., Wagner, J., Tetzlaff, A., Ingenhoven, P., Moser, D., 2014. IEA-PVPS T13-02:2014. Characterisation of performance of thin-film photovoltaic technologies.

Smith, W.L., 1985. Satellites. In: Handbook of Applied Meteorology. John Wiley \& Sons, New York (Chapter 10).
Stamnes, K., Tsay, S.-C., Wiscombe, W., Laszlo, I., 2000. DISORT, a general-purpose Fortran program for discrete-ordinate-method radiative transfer in scattering and emitting layered media: documentation of methodology Tech. rep., Department of Physics and Engineering Physics, Stevens Institute of Technology.

Stamnes, K., Tsay, S.-C., Wiscombe, W., Jayaweera, K., 1988. Numerically stable algorithm for discrete-ordinate-method radiative transfer in multiple scattering and emitting layered media. Appl. Opt. 27 (12), 2502.

Toledano, C., Cachorro, V.E., Berjon, A., de Frutos, A.M., Sorribas, M., de la Morena, B.A., Goloub, P., 2007. Aerosol optical depth and Ångström exponent climatology at El Arenosillo AERONET site (Huelva, Spain). Q. J. Roy. Meteor. Soc. 133 (624), 795-807.

Valks, P., Loyola, D., Hao, N., Rix, M., 2011. Algorithm theoretical basis document for GOME-2 total column products of ozone, minor trace gases and cloud properties. Tech. Rep. DLR/GOME-2/ATBD/01, German Aerospace Center.

Vasiliki, P., Norton, M., Hadjipanayi, M., Georghiou, G., 2013. Calibration of spectroradiometers for outdoor direct solar spectral irradiance measurements. Proc. 28th European Photovoltaic Solar Energy Conference and Exhibition, Paris, France, pp. 3466-3471.

WDC, 2015. Global ozone monitoring experiment GOME-2 https://wdc. dlr.de/sensors/gome2/ (accessed: 2015-05-25).

Weihs, P., Webb, A., 1997. Accuracy of spectral UV model calculations: 1. Consideration of uncertainties in input parameters. J. Geophys. Res. Atmos. 102, 1541-1550.

Xia, X., Chen, H., Goloub, P., Zhang, W., Chatenet, B., Wang, P., 2007. A compilation of aerosol optical properties and calculation of direct radiative forcing over an urban region in northern China. J. Geophys. Res. Atmos. 112, D12203.

Yoon, H., Gibson, C., 2011. NIST Special Publication 250-89 NIST measurement services: spectral irradiance calibrations. 